\title{
Puntos críticos del manejo poscosecha de cacao en Waslala, Nicaragua
}

\section{Critical points of cocoa postharvest management in Waslala, Nicaragua}

\begin{abstract}
Eusebio Ayestas ${ }^{1}$, Carolina Vega-Jarquín ${ }^{2 *}$, Pedro Torres ${ }^{2}$, Jurgen Lanzas ${ }^{3}$, Luís Orozco $^{4}$, Carlos Astorga $^{4}$ ${ }^{1}$ Ing. Agr. MSc. Consultor $/{ }^{2}$ Docentes investigadores UNA-FDR-FAGRO, Nicaragua $/{ }^{3}$ Lic. Agronegocio $/{ }^{4}$ CATIE-PCC, Costa Rica. (para correspondencia: carolina.vega@una.edu.ni / ayestasvillega@yahoo.es)
\end{abstract}

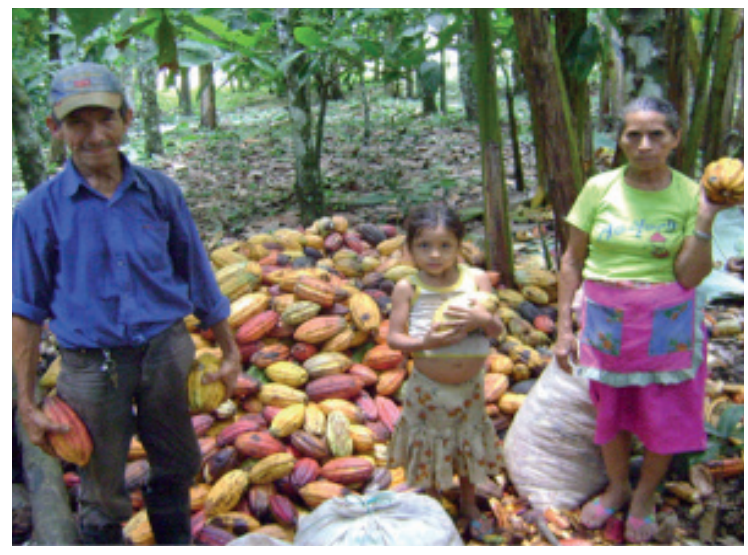

\section{RESUMEN}

En el período de enero a junio del 2009 se indagó el beneficiado de cacao en 49 fincas de productores socios de la cooperativa CACAONICA en el municipio de Waslala, Nicaragua. El propósito del estudio fue generar información de los costos, tiempos y puntos críticos del beneficiado en las fincas, y realizar un análisis FODA sobre la factibilidad de implementar el modelo de acopio de cacao en baba en dos centros comunitarios. Se aplicaron encuestas a productores y entrevistas semi-estructuradas al equipo técnico de CACAONICA y otros informantes claves vinculados con la comercialización local del cacao. Se elaboró una matriz clasificándose la información en relación a las variables tiempo, área de siembra, rendimientos y participación de la familia en la labores poscosecha del cacao. Todas las fincas visitadas poseen certificación orgánica. Treinta de los productores poseen áreas pequeñas de cacao (aproximadamente de una ha) y 19 cultivan entre 1.5 a siete ha. El beneficiado de cacao en la finca se compone de tres etapas y seis actividades: etapa 1 (cosecha y extracción-selección de semillas), etapa 2 (fermentación y secado) y etapa 3 (empacado y transporte). El proceso de beneficiado se constituye de 21.7 días calendarios, sin embargo, el tiempo real requerido en días hombre resultó ser 9.14, con un costo de USD 40.33 para beneficiar $45.45 \mathrm{~kg}$ de cacao (un qq ). La etapa 1 resultó ser la más costosa del proceso de beneficiado (USD 30.13 por cada $45.45 \mathrm{~kg}$ ). Los puntos críticos que afectan la calidad del beneficiado del grano son: fermentación (41\%) y secado $(31 \%)$. El punto de equilibrio del beneficiado en finca es $361.8 \mathrm{~kg}$ ha $^{-1}$ ciclo-1. Rendimientos por debajo de este umbral significan que el cacaotal no retribuye los costos invertidos por el agricultor. La participación de la familia en el beneficiado del cacao es relevante, especial atención requiere la participación de la mujer. La gran mayoría de los productores $(69 \%)$ perciben como buena estrategia implementar el acopio de cacao en baba en beneficios centralizados, y ratificaron su participación siempre y cuando el pago sea con-

\section{ABSTRACT}

From January to June 2009 was analyzed the post-harvest processing of cocoa in 49 cocoa producing farms members of CACAONICA cooperative, in the municipality of Waslala, Nicaragua. The purpose of the study was to generate information on costs, man hours, and critical points of postharvest processing in farms, and conduct a SWOT analysis on the feasibility of implementing a model to collect up the cocoa beans covered with mucilage in two community centers. Surveys were applied to producers and a semi-structured interview to technical team of CACAONICA, and for other key stakeholders associated with the local marketing of cocoa beans. A matrix was created to perform both the analysis and the management of variables: man hours, area planted, yields and family involvement in cocoa processing. All organic farms visited are certified. Thirty producers have small areas with cocoa, approximately $1 \mathrm{ha}$, and nineteen have between 1.5 and 7 ha. The cocoa postharvest processing into the farm consists of three stages and six activities: stage 1 (extraction- selection of seed), stage 2 (fermentation and drying) and stage 3 (packaging and transport). The process lasts 21.7 calendar days, however, the real time required in man days turned out to be 9.14 , with a total cost of USD 40.33 by $45.45 \mathrm{~kg}$ (1 quintal) of cocoa. Stage 1 was the most expensive of the postharvest processing (USD 30.13 per quintal). The critical points that affect the quality of the grain were: fermentation (41\%) and drying (31\%). The equilibrium point was $361.8 \mathrm{~kg} \mathrm{ha}^{-1}$ cycle-1, yields below this threshold means that the cacao does not pay the costs invested by the farmer. The family involvement in the postharvest processing of 
tra entrega del grano (USD 48.87 por cada $45.45 \mathrm{~kg}$ ). El éxito del nuevo sistema de acopio dependerá de la gestión de capital para el acondicionamiento de la instalación, la compra del grano a los productores, la definición de rutas lógicas de acopio en las comunidades, el equipamiento básico y la capacitación oportuna al personal técnico responsable de los centros de acopio.

Palabras clave: acopio centralizado, participación familiar, punto de equilibrio, Theobroma cacao L. cocoa is relevant, the participation of women requires more attention. Collect up the cocoa beans covered with mucilage and do a centralized postharvest processing is considered as good strategy by the vast majority of producers $(69 \%)$. They will participate as long as payment is on delivery of grain (USD 48.87 per quintal). The success of this beans collection centers will depend of the good facility conditions, the purchase of grain from farmers, the definition of routes of collecting in communities, and the equipment and training to technical personnel responsible for the collection centers.

Key words: Centralized postharvest processing, family involvement, equilibrium point, Theobroma cacao $\mathrm{L}$.

altos costos de inversión en equipamiento, tiempo y conocimiento, lo que aunado a los pocos volúmenes de cacao cosechado y fermentado, deriva en un grano que frecuentemente no cumple con la calidad requerida por el mercado. Por el contrario, un sistema de beneficiado centralizado disminuye el costo por volumen beneficiado, facilita alcanzar los estándares de calidad exigidos por el mercado debido a que los volúmenes de cacao en baba que se acopian y se fermentan son mayores, y se aplica un protocolo de fermentación con estricto control del proceso de fermentado, secado, selección y empaque, además tienen personal capacitado (Martínez et al., 2009). En este contexto, el propósito de la presente investigación fue analizar las bondades y limitantes del beneficiado de cacao en fincas de productores socios de la cooperativa CACAONICA en Waslala, para generar información base que aporte al fortalecimiento del sistema de acopio de la organización y brinde lineamientos generales para la toma de decisiones acerca de la apertura de centros de acopio comunitarios.

\section{MATERIALES Y MÉTODOS}

Descripción del sitio de estudio. El estudio se realizó en el municipio de Waslala $13^{\circ} 20^{\prime} \mathrm{N}$ y $85^{\circ} 22^{\prime} \mathrm{O}$, en la Región Autónoma del Atlántico Norte (RAAN), Nicaragua (figura 1). El municipio tiene una población de 43676 habitantes (83\% vive en el área rural y $17 \%$ en el área urbana). El 51\% de la población son mujeres. Waslala presenta características agroecológicas de la zona tropical húmedo; la altitud promedio es de $443 \mathrm{msnm}$, la humedad relativa es $84 \%$, con una temperatura media anual de $24.1^{\circ} \mathrm{C}$ y precipitación de 2750 $\mathrm{mm}$, siendo el período más lluviosos de junio a octubre. Los suelos tienen un alto contenido de arcilla, la topografía es quebrada a ondulada con pendientes promedio de $32 \%$ (Holdridge, 1979). Estas condiciones, facilitan el desarrollo de actividades agropecuarias destinadas a la producción de granos básicos (arroz, maíz, frijoles), cultivos perennes (cacao y café) y ganadería de doble propósito (Gaitán, 2005). 


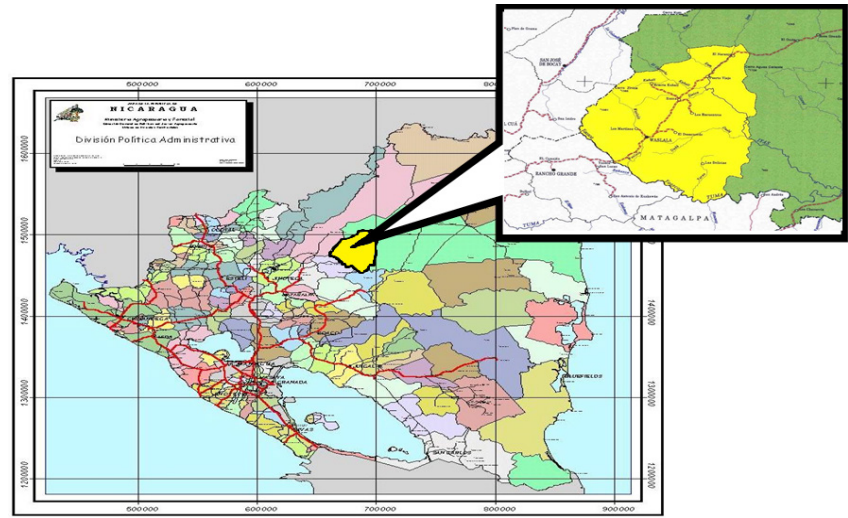

Figura 1. Mapa de Nicaragua y ubicación del municipio de Waslala.

Diseño metodológico. El estudio se realizó en 49 fincas de productores socios de CACAONICA, durante el período de enero a junio del 2009. La investigación constó de cinco etapas: (1) Diseño del estudio, se delimitó el tema, se planteó la justificación, el problema de investigación, objetivos, metodología y se establecieron las principales variables a evaluar, (2) Elaboración de instrumentos, se diseñaron encuestas, entrevistas semi-estructuradas y matrices de costos para la recopilación de datos cuantitativos (cuadro 1), (3) Validación de instrumentos, para obtener la información necesaria se realizaron entrevistas preliminares a productores con el fin de identificar las debilidades del instrumento y hacer los ajustes necesarios, (4) Etapa de campo o recolección de la información, se realizó mediante una programación de visitas a los productores y agentes comercializadores, enfatizando en la confidencialidad de la información, (5) Procesamiento de la información, se clasificó según el instrumento y se creó una base de datos en Excel para cada tipo de variable estadística (cuantitativa, cualitativa). Se codificaron las preguntas para ser registradas en su respectiva base de datos. Los datos cualitativos se analizaron en programa estadístico InfoStat 2004 y la información cuantitativa se trató con el programa Stadistics descriptive para la obtención de un razonamiento de frecuencias.

Determinación del tamaño de la muestra. Se utilizó la metodología de estratificación por racimos propuesta por Méndez (2003), adicionalmente se consideraron dos aspectos: (1) grado jerárquico en la organización y (2) el número de comunidades atendidas por CACAONICA. La fórmula aplicada se describe en el siguiente cuadro.

Cuadro 1. Descripción de los instrumentos aplicados para la recopilación de la información

\begin{tabular}{|c|c|c|c|c|}
\hline Instrumentos & Grupo meta & Cantidad & Variables evaluadas & Objetivos \\
\hline Encuestas & $\begin{array}{l}\text { Productores/as socios de } \\
\text { CACAONICA }\end{array}$ & 49 & $\begin{array}{l}\text { Descripción y grado de di- } \\
\text { ficultad del beneficiado del } \\
\text { cacao en fincas }\end{array}$ & $\begin{array}{l}\text { Conocer las dificultades logísti- } \\
\text { cas y de materiales que presenta } \\
\text { el productor al realizar el benefi- } \\
\text { ciado }\end{array}$ \\
\hline & $\begin{array}{l}\text { Equipo técnico y cuerpo di- } \\
\text { rectivo de CACAONICA. }\end{array}$ & 4 & $\begin{array}{l}\text { Capacidad logística y finan- } \\
\text { ciera }\end{array}$ & $\begin{array}{l}\text { Conocer debilidades logísticas y } \\
\text { financieras de la cooperativa }\end{array}$ \\
\hline $\begin{array}{l}\text { Entrevistas semies- } \\
\text { tructuradas }\end{array}$ & $\begin{array}{l}\text { Informantes claves (direc- } \\
\text { tivos de otras cooperativas, } \\
\text { técnico Ritter Sport, inter- } \\
\text { mediarios locales) }\end{array}$ & 3 & $\begin{array}{l}\text { Experiencias, limitaciones } \\
\text { y recomendaciones }\end{array}$ & $\begin{array}{l}\text { Conocer fortalezas y técnicas em- } \\
\text { pleadas en experiencias de siste- } \\
\text { mas de acopio }\end{array}$ \\
\hline Matriz de costos & $\begin{array}{l}\text { Productores/as socios de } \\
\text { CACAONICA }\end{array}$ & 49 & $\begin{array}{l}\text { Tiempo, costo, rendimien- } \\
\text { to, área cultivada, mano de } \\
\text { obra, manejo, equipo }\end{array}$ & $\begin{array}{l}\text { Conocer el orden lógico de las } \\
\text { actividades realizadas en el bene- } \\
\text { ficiado, tiempo y costos }\end{array}$ \\
\hline
\end{tabular}




\section{MANEETOPOSTCOSEECHA}

Cuadro 2. Descripción de la fórmula para determinar el tamaño de la muestra

\begin{tabular}{ll}
\hline \multicolumn{1}{c}{ Formula } & \multicolumn{1}{c}{ Descripción } \\
\hline $\mathrm{n} 1=\mathrm{S}^{2} / \mathrm{V}^{2}$ & $\mathrm{~N}=$ Universo $(565$ productores $)$ \\
$\mathrm{S}^{2}=\mathrm{p}(1-\mathrm{p})=0.98(1-0.98)=0.0196$ & $\mathrm{P}=$ Probabilidad $(98 \%)$ \\
$\mathrm{V}^{2}=(\mathrm{E}) 2=(0.02) 2=0.0004$ & $\mathrm{E}=$ Error $(2 \%)$ \\
$\mathrm{n}=\mathrm{n} 1 / 1+\mathrm{n} 1 / \mathrm{N}$ & $\mathrm{n} 1=$ muestra sin ajustar \\
$\mathrm{n}=49 / 1+49 / 565$ & $\mathrm{~S}^{2}=$ nivel de probabilidad verdadera \\
$\mathrm{n}=49$ & $\mathrm{~V}^{2}=$ nivel de probabilidad en error \\
& $\mathrm{n}=$ Tamaño de la muestra \\
\hline
\end{tabular}

Tipología de los productores. Todos los productores visitados cultivan cacao de manera orgánica y están certificados por Biolatina. El universo de productores evaluados se clasifican en tres grupos según la superficie cultivada: pequeños -30 productores- con áreas entre 0.35 y 1.40 ha, medianos -13 productores- que poseen desde 1.41 hasta 2.81 ha y grandes -6 productores- que cultivan de 2.82 a 7.04 ha.

Manejo agronómico de los cacaotales. Los cacaotales son establecidos a distancias de 4 x 4 m (625 plantas ha- $\left.{ }^{-1}\right)$, los productores realizan tres podas al año (mayo, agosto y noviembre), la poda de mantenimiento se realiza en mayo, con el inicio de la época lluviosa. El 75\% de los cacaotales presentaron diseños de sombra compuesto por, 1) frutales y 2) especies maderables. El 90\% de los productores realizan un manejo de arvenses de manera manual (machete); esta se realiza tres veces al año. La prevención y manejo de enfermedades se efectúa cortando y enterrando los frutos enfermos con intervalos de ocho días, además aplican productos elabora- dos en la finca, tales como cal disuelta en agua aplicada a los frutos pequeños (pepinos) y cal en polvo depositada alrededor del tallo del árbol. La fertilidad del suelo se mejora aplicando biocompost y fertilizante foliar preparado con material producido en la finca. El material genético predominante en las fincas es Trinitario, empleándose la técnica de injertación para propagar y fomentar sus cacaotales (Ayestas, 2009).

Etapas, actividades y costos del beneficiado de cacao en fincas. El beneficiado de cacao en fincas, se compone de tres etapas y seis actividades principales. La etapa 1 representa las actividades de cosecha, quiebre de mazorcas y extracción de las semillas; esta etapa resultó ser la más costosa por el uso de mano de obra externa al hogar (USD 30.13 - 6.85 $\mathrm{D} / \mathrm{H}$ ) (cuadro 3). Para realizar todo el proceso de beneficiado, los productores necesitan $9.14 \mathrm{D} / \mathrm{H}$ y una inversión de USD 40.33 en el beneficiado de $45.45 \mathrm{~kg}$ de cacao (un quintal).

Cuadro 3. Etapas y costos del proceso de beneficiado de $45.45 \mathrm{~kg}$ (un quintal) de cacao en Waslala, Nicaragua

\begin{tabular}{|c|c|c|c|}
\hline Etapas y actividades & $\begin{array}{l}\text { Duración total del proceso } \\
\text { en días calendarios }\end{array}$ & Tiempo requerido $\mathrm{D} / \mathrm{H}$ & $\begin{array}{l}\text { Costo por actividad } \\
\text { (USD) }\end{array}$ \\
\hline \multicolumn{4}{|l|}{ Etapa I } \\
\hline Cosecha & 3.59 & 3.59 & 16.15 \\
\hline Recolecta & 0.55 & 0.55 & 2.47 \\
\hline Traslado al lugar de extracción & 0.55 & 0.55 & 2.47 \\
\hline Extracción y selección de semillas & 1.90 & 1.90 & 7.86 \\
\hline Traslado a los fermentadores & 0.26 & 0.26 & 1.19 \\
\hline Sub-total & 6.85 & 6.85 & 30.13 \\
\hline \multicolumn{4}{|l|}{ Etapa II } \\
\hline $\begin{array}{l}\text { Fermentación (tiempo dedicado al pro- } \\
\text { ceso) }\end{array}$ & 8.00 & 0.45 & 2.02 \\
\hline Secado (dedicado al proceso) & 6.20 & 1.19 & 5.37 \\
\hline Sub-total & 14.20 & 1.64 & 7.39 \\
\hline \multicolumn{4}{|l|}{ Etapa III } \\
\hline Empaque & 0.18 & 0.18 & 0.80 \\
\hline Transporte & 0.47 & 0.47 & 2.01 \\
\hline Sub total & 0.65 & 0.65 & 2.81 \\
\hline TOTAL & 21.7 & 9.14 & USD 40.33 \\
\hline
\end{tabular}

*Tipo de cambio (USD 1= C\$ 20.15) - Costo D/H (USD 4.46). 
Puntos críticos del beneficiado de cacao en fincas. La mayoría de productores -36- mencionaron tener dificultades con las actividades de fermentado y secado. Argumentan no poseer la herramienta y la técnica para medir la temperatura y evaluar las características externas e internas de las semillas en el momento de realizar las pruebas de corte durante el desarrollo del proceso del fermentado. Otro aspecto señalado fue el poco volumen acopiado y fermentado, el cual no permite alcanzar la temperatura requerida para una buena fermentación, afectando la calidad del grano. La inestabilidad del clima (abundantes lluvias y humedad relativa que varía entre 70 a $80 \%$ ) es otro factor que dificulta el secado y almacenamiento del grano (cuadro 4). Asimismo, identificaron como punto crítico el transporte, principalmente las condiciones higiénicas para el traslado del grano desde la finca hasta el centro de acopio de CACAONICA en Waslala. Por otro lado, se determinó que las plantaciones de cacao de cinco productores tienen problemas por enfermedades y que mezclan mazorcas enfermas, inmaduras, dañadas y de diferentes variedades en la misma masa de fermentación.

Cuadro 4. Puntos críticos del proceso de beneficiado de cacao en las fincas de Waslala, Nicaragua

\begin{tabular}{cc}
\hline Puntos críticos por actividad & $\begin{array}{c}\text { Cantidad de productores con } \\
\text { problemas }\end{array}$ \\
\hline Cosecha & 10.20 \\
Extracción & 0.00 \\
Fermentado & 40.82 \\
Secado & 32.65 \\
Empacado & 4.08 \\
Trasporte & 12.24 \\
\hline
\end{tabular}

Punto de equilibrio del beneficiado del cacao en las fincas. Los resultados demuestran que el punto umbral o de equilibrio de volumen es $361.8 \mathrm{~kg} \mathrm{ha}^{-1}$ por ciclo (7.96 qq ha ${ }^{-1}$ ci$\mathrm{clo}^{-1}$ ), lo que se traduce considerando el precio de venta en un ingreso monetario de 1184 dólares. Es decir, para que el cacaotal retribuya al productor la inversión en tiempo y dinero, éste debe alcanzar volúmenes e ingresos similares o mayores a los indicados (figura 2). De lo contrario, si la cosecha del ciclo es inferior a $361.8 \mathrm{~kg} \mathrm{ha}^{-1}$, el agricultor experimentará pérdida de dinero y su tiempo no será compensado.

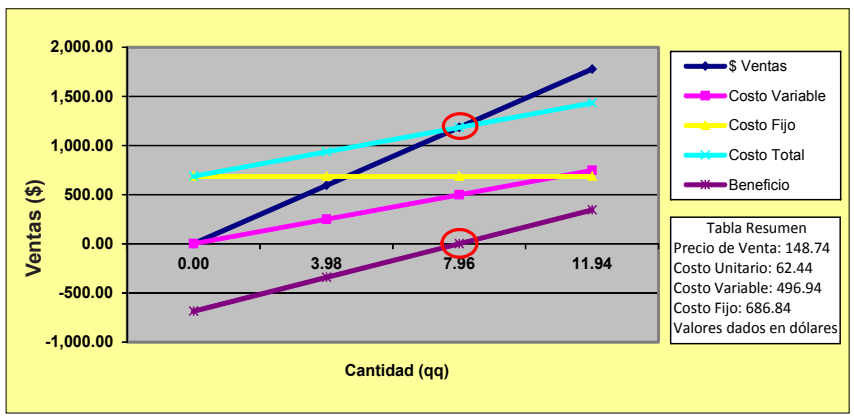

Figura 2. Punto de equilibrio del beneficiado de cacao en fincas, Waslala, Nicaragua. Sacos de $45.45 \mathrm{~kg}=1$ unidad de quintal.
Participación familiar en el beneficiado del cacao en las fincas. Al analizar las tres etapas del beneficiado (cuadro 5) y la participación familiar, se identifica que en las tres etapas, el miembro más involucrado es el jefe de familia. Sin embargo, en la tercera etapa la contratación de mano de obra externa se incrementa, debido al requerimiento de fuerza física ya que esta etapa se compone del empacado y traslado del producto a la cooperativa. Además, es importante destacar la participación de la mujer en las dos primeras etapas, involucrándose en un 35 y $40 \%$, en su orden. Las actividades que contemplan estas dos etapas (cuadro 3), expresa la responsabilidad asumida por las mujeres, ya que de ellas depende en gran medida la calidad final del grano de cacao.

Cuadro 5. Participación familiar (\%) en el beneficiado del cacao en fincas de productores, Waslala, Nicaragua

\begin{tabular}{lccc}
\hline Integrante & Etapa 1 & Etapa 2 & Etapa 3 \\
\hline Jefe de familia & 45 & 43 & 38 \\
Esposa & 35 & 40 & 10 \\
Hijo & 30 & 36 & 27 \\
Contratación & 8 & 13 & 35 \\
Otros & 12 & 5 & 0 \\
\hline
\end{tabular}

Percepción del acopio en baba, precio de venta y formas de pago en centros de acopios. El $69.39 \%$ de los productores están dispuestos a vender el cacao en baba, el restante $30.61 \%$ afirmó continuar beneficiando el cacao en sus fincas, debido a dificultades en las vías de acceso a sus comunidades.

Con respecto al precio de venta este oscila entre 48 y 79.41 dólares, sin embargo, en promedio a los productores les gustaría haber recibido al menos en USD 48.87 por cada $45.45 \mathrm{~kg}$ de cacao en baba, al considerar que posiblemente este precio les permitiría sufragar los costos de manejo del cacaotal. Con relación a la forma de pago, el $65 \%$ de productores encuestados proponen a la cooperativa realizar el pago en efectivo de manera inmediata, mientras que un $33 \%$ están dispuesto a recibir su pago en cheque.

Cuadro 6. Disposición de los productores para la venta de cacao en baba en CACAONICA, Waslala, Nicaragua

\begin{tabular}{lc}
\hline \multicolumn{2}{c}{ Precio de venta (USD) por cada $45.45 \mathrm{~kg}$ de cacao en baba } \\
\hline Precio promedio & 48.87 \\
Precio mínimo & 29.78 \\
Precio máximo & 79.41 \\
\hline \multicolumn{2}{c}{ Porcentaje de opinión sobre formas de pago } \\
\hline \multirow{2}{*}{ Efectivo } & 65 \\
Cheque & 33 \\
Recibo & 2 \\
\hline
\end{tabular}

* Tipo de cambio: USD 1 = C\$20.15. 
Análisis FODA y visión compartida de un nuevo sistema de acopio centralizado. Las entrevistas realizadas al técnico de la cooperativa CACAONICA, junta directiva, técnico de Ritter Sport y productores, permitió el análisis FODA para el funcionamiento efectivo del nuevo sistema de acopio comunitario de cacao en baba.

Etapas, actividades del beneficiado y punto de equilibrio. El beneficiado del cacao es una de las etapas que determina la calidad de la almendra. En general, el beneficiado consta de seis actividades: cosecha, desgrane, fermentado, secado, almacenado y trasporte (Cros, 2009; Büchert, 2008; Navarro, 2010), que generan costos variables según la localidad. En este estudio se estimaron costos más bajos y requerimiento de menos días hombre para realizar las seis actividades en relación a los reportados por productores de Río San Juan (USD 67.74 y $12.5 \mathrm{D} / \mathrm{H}$ (Navarro, 2010). De las seis actividades los productores consideraron, el fermentado y secado como los puntos críticos.

Se considera que la fermentación y secado son procesos que limitan el alcance de la calidad óptima de la almendra de cacao. Superar estas limitantes en el proceso de beneficiado del cacao implica realizar cambios en las prácticas de manejo de poscosecha e invertir en equipos y en infraestructura, lo que significa un desafío en el control de la calidad del cacao en Nicaragua (Cros, 2009; Villalobos y Orozco, 2009; Martínez et al., 2009; Büchert, 2008).

El punto de equilibrio establecido en este estudio (361.8 $\mathrm{kg} \mathrm{ha}^{-1}$ año $\left.^{-1}\right)$ está por

\section{RESULTADOS Y DISCUSIÓN}

Tipología de los productores. En Nicaragua, el sector productivo cacaotero en su mayoría está conformado por pequeños productores que habitan y trabajan en zonas de difícil acceso, ubicadas generalmente dentro de áreas protegidas de interés nacional. Es por eso que la distribución de los cacaoteros coincide con el Corredor Biológico Mesoamericano del Atlántico (CBMA). El área de cacao cultivada por productores que establecen en promedio 1 ha por finca representa el $74.8 \%$ del total a nivel nacional (Orozco y Deheuvels, 2007; OXFAM, 2008).

Los productores nicaragüenses tradicionalmente han asociado el cacao con árboles frutales que asegura y diversifica la dieta alimenticia de la familia, así como con especies maderables que brindan sombra y son aprovechados para generar ingresos extras al núcleo familiar (CATIE, 2010; Ayestas, 2009; Büchert, 2008; OXFAM, 2008; Villalobos y Orozco, 2009). Este escenario, es similar en los demás países de la región centroamericana, los cuales comparten el paradigma de agricultura de subsistencia (Orozco y Deheuvels, 2007). encima del rendimiento promedio nacional $\left(236 \mathrm{~kg} \mathrm{ha}^{-1}\right.$ año $o^{-1}$ y del rendimiento promedio en Waslala $\left(328 \mathrm{~kg} \mathrm{ha}^{-1}\right.$ año ${ }^{-1}$ ), lo que refleja que los productores no obtienen retribución monetaria del cultivo de cacao y que los costos referentes a manejo y cosecha no han sido sufragados por los ingresos de la venta del cacao. Esto se traduce en una nula o mínima capitalización anual (Villalobos y Orozco, 2009; CATIE, 2009). Sin embargo, por el tipo de diseño agroforestal en que se cultiva el cacao (SAF-cacao), la diversificación dentro de estos sistemas pueden significar un aporte económico sumatorio y de seguridad alimentaria a las familias dedicadas al rubro cacao (Sáenz, 2012).

Partición familiar, percepción del sistema de acopio y análisis FODA. Por su tipología de pequeños productores y por sus características económicas, la mayoría se ven forzados a emplear la fuerza laboral familiar (Sáenz, 2012; OXFAM 2008). Los datos demuestran que el involucramiento de la familia es significativo en las actividades productivas del cacao. De manera que las familias de Waslala que culti- 
van cacao emplean manejo de subsistencia, planificando y definiendo las actividades donde requieren contratar mano de obra, dado que el cacao no les genera ingresos suficientes que les permita contratar personal en las demás actividades, optando, por emplear a los miembros de la familia y de esta manera tratar que el ingreso por cacao quede en manos del núcleo familiar, el cual servirá para sufragar gastos del hogar.

Montoya (2009) reportó que la participación de la mujer es relevante en las labores de poscosecha (fermentación y secado). En Nicaragua el aporte de las mujeres rurales a la economía de sus hogares es del $32 \%$ y su participación en las actividades agrícolas productivas es muy variable y aumenta generalmente con el grado de pobreza, en ocasiones llega a representar más del $50 \%$ de la mano de obra, lo que evidencia que los sistemas productivos son mixtos y no expresamente masculinos (Ballara et al., 2010).

Fernández (2006) declara que la aceptación por la implementación de un sistema de acopio centralizado, depende del acceso vial a cada comunidad, por lo que los productores que habitan en comunidades remotas y con mal acceso estarán más renuentes a vender su cacao en baba, debido a la pérdida de las propiedades químicas durante el transcurso al centro de acopio y que finalmente afectaría la calidad del grano. La mayoría de los productores solicitan que se les cancele su cacao contra entrega, sin embargo, dependerá en gran medida del flujo de caja que posea la organización, por lo tanto, lo más sensato para la cooperativa será cancelar de forma inmediata y en efectivo al momento que el productor entrega su cacao en los centros de acopios comunitarios (Martínez et al., 2009).

\section{CONCLUSIONES Y RECOMENDACIONES}

El proceso de beneficiado está compuesto por tres etapas y seis actividades principales: etapa 1 (cosecha y desgrane), etapa 2 (fermentación y secado), etapa 3 (almacenamiento y transporte). La etapa 1, es la más costosa para el pro- ductor (USD 30.13), en cambio, la fermentación y secado, fueron consideradas como puntos críticos del beneficiado.

El tiempo y recursos requerido para beneficiar $45.45 \mathrm{~kg}$ (un qq) de cacao en la finca es de 9.14 días hombre de 21.7 días calendarios establecidos y 40.33 dólares americanos.

El punto de equilibrio se estableció en $361.8 \mathrm{~kg} \mathrm{ha}^{-1}$ ciclo $^{-1}$, lo que significa que los rendimientos de los cacaotales deben ser superiores a este umbral para generar utilidades, de lo contrario, significará pérdida para el productor. Se determinó que la participación femenina en el proceso de beneficiado es de $35 \%$ en la etapa I y $40 \%$ en la etapa II.

La cooperativa deberá mantener un fondo de liquidez para los centros de acopio comunales, destinado a la compra del cacao en baba. Así mismo, disponer de una ruta de acopio eficiente, que permita la organización y programación de entrega del cacao en baba por parte de los productores en las comunidades.

Para la ejecución del sistema de acopio en centros comunales se deberán diseñar normas, procedimientos del proceso, y coordinación adecuada. Por otro lado, además de dotarlos de herramientas y equipos, es crucial realizar capacitaciones en las actividades de beneficiado centralizado y gestión empresarial al personal que se asigne para atender estos centros.

\section{AGRADECIMIENTOS}

A Bioversity y al proyecto NICACAO por el apoyo financiero a esta investigación. Al personal y promotores de CACAONICA-PCC, en particular a Ing. Aldo Kuant, Jorge Aguilar técnicos de los proyectos NICACAO y PCC, por el apoyo brindado durante la etapa de campo. Al Ing. Miguel Malespín por sus valiosos comentarios. A los productores de Waslala por su hospitalidad y colaboración. Al representante de PRO-MUNDO HUMANO, Lic. Hans Grebe y al personal de OTN-Nicaragua por la atención y el apoyo administrativo.

\section{REFERENCIAS BIBLIOGRÁFICAS}

Ayestas V, E. 2009. Caracterización morfológica de cien árboles promisorios de cacao (Theobroma cacao L), en Waslala, RAAN, Nicaragua. Tesis Ing. Agrónomo. Universidad Nacional Agraria, Managua, NI. 57 p.

Ballara, M; Damianovic, N; Parada, S. 2010. Aporte de ingreso económico de las mujeres rurales a sus hogares. Fundación Latinoamericana de innovación social. Santiago, CL. 69 p.

Büchert, JP. 2008. Nicaragua: Agrocadena de cacao sostenible y comercio justo. II foro nacional del cacao Managua-NI. 11 p.

CATIE (Centro Agronómico Tropical de Investigación y Enseñanza). 2010. Gestión del conocimiento en el sector cacaotero mundial: conceptos y experiencias en Nicaragua. Serie foros técnicos: Modernización de la cacaocultura de Centroamérica. Managua, NI, 41 p. 2009. Proyecto competividad y ambiente en los territorios cacaoteros de Centroamérica. Informe trimestral abril a junio. 71 p.

Cros, E. 2009. Calidad integral del cacao. Serie foros técnicos: Modernización de la cacaocultura de Centroamérica. Foro II: El cacao fino de aroma en la estrategia cacaotera de Nicaragua. Presentación. CIRAD.

Fernández, R. 2006. Estudio de factibilidad para el establecimiento de un sistema de comercialización basado en rutas de acopio de cacao para la cooperativa agroforestal y comercialización de cacao (CACAONICA). Pro-Mundo Humano. Managua. 30 p.

Gaitán, T. 2005. Cadena del cultivo de cacao (Theobroma Cacao L.) con potencial exportador. Managua. Nicaragua. 60 p.

Holdridge, PL. 1979. Ecología basada en zonas de vida. Ed. IICA, San José, CR. 159 p. 


\section{QMANEDOPOSTCOSEECHA}

Malespín, M. 2010. Cosecha, beneficiado y calidad del cacao. Diplomado Cacaocultura Moderna. Presentación. Programa fomento de cacao PPP DeD/ADDAC/Ritter.

Martínez, T; Navarro, M; Camacho, A. 2009. Cacao de calidad beneficiado en centros de acopio: Manual paso a paso. ProDeSoC-IPADE. 23 p.

Méndez, C. 2003. Metodologia. 2da. ed. McGraw-Hill. Bogotá, CO. 169 p.

Montoya, J. 2009. Posicionamiento y gobernanza de organizaciones de productores en cadenas de valor globales: el caso de la cooperativa nicaragüense CACAONICA. Tesis Magíster Scientiae en socio economía. CATIE, Turrialba, CR. 162 p.

Navarro, M. 2010. Beneficiado del cacao en fincas versus centros de acopio: competitividad económica y calidad. Diplomado Cacaocultura moderna. Presentación. Mesa Nacional del Cacao.

Orozco, L; Deheuvels, O. 2007. El cacao en Centroamérica: resultados del diagnóstico de familias, fincas y cacaotales. Informe final de diagnóstico. Proyecto Cacao Centroamérica (PCC). CATIE, Turrialba, CR. 148 p.

OXFAN (Oxford Commitee for Famine Relie, UK). 2008. Análisis de cadena y mercado para Lácteos y Cacao: Triángulo Minero, RAAN. Managua, NI. 192 p.

Rodríguez, N. 2006. Beneficio del cacao (Theobroma cacao L.). Universidad Central de Venezuela, Departamento e instituto de agronomía. 32 p.

Sáenz, Y. 2012. Aporte del cacao en la economía y nutrición familiar en Waslala, Nicaragua. Tesis MSc. CATIE, Turrialba, CR. 73 p. Villalobos, M; Orozco, S. Comps. 2009. Calidad de cacao en Centroamérica: un vistazo a la situación 2009. Turrialba, Costa Rica, CATIE. 90 p. 